\title{
Retention in Care, Loss to Follow-up and Associated Patient Characteristics: A Retrospective Cohort Study among Adults Receiving Antiretroviral Therapy from Urban Health Facilities in Ghana
}

\author{
Raphael Kweku Obeng ${ }^{1 *}$, Berko Panyin Anto ${ }^{1}$, Joseph Attakorah ${ }^{1}$, Ebenezer Frimpong ${ }^{2}$ \\ and Richard Morgan ${ }^{2}$ \\ ${ }^{1}$ Department of Pharmacy Practice, Kwame Nkrumah University of Science and Technology, Ghana \\ ${ }^{2}$ Directorate on Medicine, Komfo Anokye Teaching Hospital, Ghana
}

\begin{abstract}
Introduction: Assessing treatment outcomes among persons receiving antiretroviral therapy (ART) for human immunodeficiency virus (HIV) infection is important for clinical and public health decisions. Although Komfo Anokye Teaching Hospital and Suntreso Government Hospital have offered ART to several patients for more than a decade, their treatment outcomes have not been well reported. We aimed at investigating retention in care, loss to follow-up, and patient characteristics associated with these outcomes among HIV-infected adults receiving ART from two urban treatment sites in the Ashanti Region of Ghana in order to design interventions to improve therapy outcomes.

Materials and method: We conducted medical records review (MRR) from September to December 2020 among HIVinfected patients receiving ART from two urban ART sites in Kumasi, Ghana. Patients were eligible if they were adults aged $\geq 20$-years and had taken ART for $\geq 3$-years as of December 2019. Retention in care, loss to follow-up (LTFU) and mortality among our study patients were investigated from ART initiation up to 3-years. In this study, retention of patients in care was defined as actively attending clinic and picking ARVs within the follow-up period. Loss to follow-up was defined as having discontinued clinic attendance and ARVs pick-up for more than 90 days after the last scheduled visit. Logistic regression analyses were performed to study the treatment outcomes and their associated patient characteristics.
\end{abstract}

Results: Of the 370 patients whose medical records were reviewed, 258 (69.7\%) were female, 260 (70.3\%) were retained in ART up to 3-years, $29.7 \%$ were lost to follow-up of whom $9.1 \%$ were confirmed dead. Overall mortality was therefore 2.7\%. Being employed (AOR 2.55, $p=0.015,95 \% \mathrm{Cl} 1.201-5.4 .414$ ), female (AOR 1.319, Cl 95\% 0.754-2.310), divorced (AOR 1.266, Cl 95\% 0.506-3.166) and having primary level of education (AOR 1.539, Cl 95\% 0.915-2.590) were associated with greater odds of being retained in care.

Conclusion: Although mortality among our study cohort was low and bout 70\% were retained in ART up to 3-years, loss to follow-up was high, especially within the first 6 months. Effective follow-up interventions are required to reduce LTFU during the first 6 months of treatment and among younger adults, patients with secondary and tertiary education as well as single and married patients who were less likely to be retained in care.

\section{Keywords}

Antiretroviral therapy, Treatment outcomes, Retention in care, Loss to follow-up, Ashanti Region, Ghana

\section{Introduction}

The efficacy of antiretroviral medicines (ARVs) for prolonging life of persons living with human immunodeficiency virus (HIV) infection has not been in doubt [1]. As the world continues to await a cure for HIV infection, ART remains a life life-long venture but retention in antiretroviral therapy (ART) programs has been a significant challenge to persons receiving ART $[2,3]$. Many large and small studies have reported ART outcomes such as survival or retention in care, loss to follow-up, mortality as well as immunologic and virologic response to ART. Previous studies have reported overall treatment retention rate of $65 \%$ within 2-4-years of ART $[4,5]$ and $86 \%$ after 4 -years of ART [6]. A study of large data from 42 countries has estimated retention in ART at month 36 , to be $68 \%$, with regional retention rate of $65 \%$ for Africa, $80 \%$ for Asia, $64 \%$ for Latin America and the Caribbean [5]. Meta-analysis of large data from 45 studies in Ethiopia 
Citation: Obeng RK, Anto BP, Attakorah J, et al. (2021) Retention in Care, Loss to Follow-up and Associated Patient Characteristics: A Retrospective Cohort Study among Adults Receiving Antiretroviral Therapy from Urban Health Facilities in Ghana. Clin J HIV AIDS 5(1):70-75

has revealed that pooled magnitude of retention in clinical care was $70.65 \%$, with overall magnitude of loss to follow-up (LTFU) being $15.17 \%$ [7].

Other researchers have reported among a Ghanaian cohort that after 3-years of follow-up, overall LTFU from ART was $31 \%$, with $67 \%$ of such cases occurring within the first 12 months of treatment [8]. It has been determined that independent predictors of attrition from ART programs include male sex, being younger adult, single, divorced or separated [9]. According to Alvarez, et al. 2013, being female was associated with reduced risk of attrition but being single, illiterate and aged < 25-years-old were associated with increased risk of LTFU [10]. Studies from sub-Saharan Africa show that the cumulative incidence of attrition from ART programs can be up to 35\% in 3-years of follow-up [5].

Regarding ART-related mortality, analyses of 18 published cohort studies have revealed that between $8 \%$ and $26 \%$ of patients offered ART in sub-Saharan Africa die within the first year of treatment [11], with $14 \%$ dying within the first 3 months [4]. Some other researchers have reported that ART-associated rates of mortality during the initial stages of treatment were higher in low income settings $[5,12]$. Assessing ART outcomes among persons living with HIV is therefore important for clinical and public health decisions but results of ART outcomes of our study sites have not been previously reported. We aimed at investigating retention in ART, loss to follow-up, mortality and patient characteristics associated with these outcomes among patients receiving ART from two urban ART programs in Ghana in order to design interventions to improve treatment outcomes.

\section{Materials and Method}

\section{Study setting and population}

The study was conducted at two antiretroviral treatment sites in the Ashanti Region of Ghana namely Komfo Anokye Teaching Hospital (KATH) and Suntreso Government Hospital (SGH) both in Kumasi. The study sites are similar in operations since they use national treatment guidelines and receive supplies of antiretroviral medicines and other logistics from government without cost to the individual facilities and patients. The two sites are located approximately $2.5 \mathrm{~km}$ apart in the second largest city of Ghana and therefore have urban characteristics. As a teaching hospital, KATH receives patients from different parts of the country (including persons living with HIV/AIDS) through a referral system. As part of its clinical services, SGH runs sexually transmitted infections (STI) clinic where patients who test positive for HIV are offered ART. The study population was made up of HIV-infected adults aged $\geq$ 20-years who were receiving ART as at December 2019. To qualify for inclusion, patients were additionally expected to have taken ART for $\geq 3$-years and had the required information in their medical records for review. Patients who initiated ART in other facilities but were transferred to the study sites were excluded from the study.

\section{Sample size estimation}

We used sample size calculation formula recommended by Bill Godden 2004 [13], with input from Rose, Spinks \& Canhoto, 2015 [14] to calculate required sample size from an estimated population of 7,200 HIV-infected adults receiving clinical care from the two facilities. The minimum sample size required was 365 folders but in order to make room for any possible data insufficiency from some of the records the number of folders to be reviewed was increased to 385 .

\section{Data collection process}

A data collection tool designed in Microsoft excel was used to extract selected patients' details from their hospital records. Quota random sampling technique was used to apportion the number of folders to be reviewed from each study site. The apportioned number of folders for each study site was collected from patients who initiated treatment in the year 2014, 2015, 2016 and 2017. We conducted medical records review (MRR) among our study patients to investigate patient retention in care, loss to follow-up, mortality and patient characteristics such as age, gender, educational, marital and occupational background associated with these outcomes. Within the age category we divided the patients into three groups described as young adults (20-40-years), middleaged adults (41-60-years) and older adults (61-80-years). For each participant, the required data were extracted to cover a period of 3-years starting from the day of ART initiation. For patients who died or were lost to follow-up in the course of treatment, their data were extracted from the day of ART initiation to the last date of clinic attendance. Data collection was done between September and December 2020.

In this study retention of patients in ART was defined as actively attending clinic and refilling ARVs within the followup period. Loss to follow-up of a patient was defined as having discontinued clinic attendance and ARVs pick-up for more than 90 days after the last scheduled visit. Patients who defaulted treatment for any length of time but had returned to continue ART within the 3 years of follow-up were considered to be retained in care.

\section{Data management and analysis}

Participants' data collected from the two study sites were aggregated and entered into Stata version12 software for analysis. Descriptive statistics were carried out on variables such as age, gender, marital, occupational and educational backgrounds of study patients in relation to their treatment outcomes such as retention in care, loss to follow-up and

*Corresponding author: Raphael Kweku Obeng, Faculty of Pharmacy and Pharmaceutical Sciences, Department of Pharmacy Practice, Kwame Nkrumah University of Science and Technology, Kumasi, Ghana, Tel: +233244465082

Accepted: November 17, 2021

Published online: November 19, 2021

Citation: Obeng RK, Anto BP, Attakorah J, et al. (2021) Retention in Care, Loss to Follow-up and Associated Patient Characteristics: A Retrospective Cohort Study among Adults Receiving Antiretroviral Therapy from Urban Health Facilities in Ghana. Clin J HIV AIDS 5(1):70-75 
Citation: Obeng RK, Anto BP, Attakorah J, et al. (2021) Retention in Care, Loss to Follow-up and Associated Patient Characteristics: A Retrospective Cohort Study among Adults Receiving Antiretroviral Therapy from Urban Health Facilities in Ghana. Clin J HIV AIDS 5(1):70-75

Table 1: Patient characteristics, retention in care and loss to follow-up from urban ART program in Ghana.

\begin{tabular}{|c|c|c|c|}
\hline $\begin{array}{l}\text { Patient } \\
\text { Characteristics }\end{array}$ & $\begin{array}{l}\text { Patient } \\
\text { Category }(\mathrm{N}=370)\end{array}$ & Retention in care & Loss to follow-up \\
\hline \multirow[t]{2}{*}{ Gender } & Female 258 (69.3\%) & $188(72.9 \%)$ & $70(27.1 \%)$ \\
\hline & Male 112 (30.7\%) & $72(64.3 \%)$ & $40(35.7 \%)$ \\
\hline \multirow{3}{*}{$\begin{array}{l}\text { Age Group } \\
\text { (Years) }\end{array}$} & $21-40200$ (54.1\%) & $135(67.5 \%)$ & $65(32.5 \%)$ \\
\hline & $41-60160(43.2 \%)$ & $118(73.8 \%)$ & $42(26.2 \%)$ \\
\hline & $61-8010(2.7 \%)$ & $7(70.0 \%)$ & $3(30.0 \%)$ \\
\hline \multirow[t]{2}{*}{ Occupation } & Employed 333 (90\%) & $240(72.1 \%)$ & $93(27.9 \%)$ \\
\hline & Unemployed 37 (10\%) & $20(54.1 \%)$ & $17(45.9 \%)$ \\
\hline \multirow[t]{4}{*}{ Education } & Primary 199 (53.8\%) & $150(75.4 \%)$ & $49(24.6 \%)$ \\
\hline & Secondary 28 (7.6\%) & $16(57.1 \%)$ & $12(42.9 \%)$ \\
\hline & Tertiary 16 (4.3\%) & $8(50 \%)$ & $8(50 \%)$ \\
\hline & Uneducated 127 (34.3\%) & $86(67.7 \%)$ & $41(32.3 \%)$ \\
\hline \multirow[t]{4}{*}{ Marital status } & Divorced 72 (19.5\%) & $60(83.3 \%)$ & $12(16.7 \%)$ \\
\hline & Married 146 (39.5\%) & $91(62.3 \%)$ & $55(37.7 \%)$ \\
\hline & Single $89(24.1 \%)$ & $59(66.3 \%)$ & $30(33.7 \%)$ \\
\hline & Widowed 63 (17.0\%) & $50(79.4 \%)$ & $13(20.6 \%)$ \\
\hline \multirow[t]{4}{*}{ ART initiation Regimen } & AZT/3TC/EFV 88 (23.8\%) & $54(61.4 \%)$ & $34(38.6 \%)$ \\
\hline & AZT/3TC/NVP 109 (29.5\%) & 79 (72.5\%) & $30(27.5 \%)$ \\
\hline & TDF/3TC/EFV 114 (30.8\%) & $86(75.4 \%)$ & $29(26.4 \%)$ \\
\hline & TDF/3TC/NVP 59 (15.9\%) & $42(71.2 \%)$ & $17(28.8 \%)$ \\
\hline
\end{tabular}

Note: TDF is tenofovir disoproxyl fumarate, AZT is azidothymidine (zidovudine), 3 TC is lamivudine, NVP is nevirapine and EFV is efavirenz

mortality. Multivariate analysis was done using logistic regression to investigate the level of significance and associations between variables and treatment outcomes using odds ratios, $p$-values of $<0.05$ and $95 \%$ confidence interval.

\section{Results}

\section{Characteristics of study patients}

The number of folders selected to be reviewed was 385 but 15 were rejected due to data insufficiency leaving a total of 370. The mean age of patients was 40.4-years (SD 9.29). Females constituted $69.7 \%(n=258)$ of the 370 patients whose medical records were reviewed. Majority of the study patients $54.1 \%(n=200)$ were $21-40$ years-old, followed by $43.2 \%(n=160)$ in the $41-60$ years age group. Altogether the two age groups constituted $97.3 \%$ (Table 1 ). While $90 \%$ $(n=333$ ) of the study patients were employed, 37 were unemployed. Approximately $88 \%$ of the study patients were either uneducated or had primary level of education. Only $12 \%$ had either secondary or tertiary education. While $39.5 \%$ were married, the remaining study patients were divorced (19.5\%), single $(24.1 \%)$ or widowed $(17.0 \%)$.

\section{Retention in care and loss to follow-up from ART}

Of the 370 study patients whose medical records were reviewed, 260 (70.3\%) were retained in care up to 3-years but 110 (29.7\%) were lost to follow-up at various times within the period (Table 1). Among the 258 females whose records were reviewed, $27.1 \%(n=70)$ were lost to follow-up while $35.7 \%(n=40)$ of the males were also lost to follow-up. For patients aged $21-40$ years described as younger adults, LTFU was $32.5 \%$ which was relatively higher than that of patients in the middle age group of $41-60$ years with $26.2 \%$ LTFU. Although only $10 \%$ of the study patients were unemployed, LTFU of approximately $46 \%$ was recorded among this group as opposed to $27.9 \%$ LTFU recorded among the employed patients. Considering marital status, LTFU was high among married clients (37.3\%) than those who were not married (single, divorced and widowed). The lowest LTFU rate of $16.7 \%$ was recorded among divorced clients followed by 20.6\% for widowed patients (Table 1 ).

From logistic regression analysis results in Table 2, only employment status of study patients had statistically significant association with being retained in care. Employed patients (AOR 2.55, $p=0.015,95 \% \mathrm{Cl} 1.201-5.4 .414$ ) had greater odds of being retained in care as compared to unemployed patients. The results however show that being female (AOR 1.319, Cl 95\% 0.754-2.310), divorced (AOR $1.266, \mathrm{Cl} 95 \% 0.506-3.166)$ and having primary education were associated with greater odds of being retained in care. Conversely, being younger adult with age 21-40-years (AOR $0.767, \mathrm{Cl} 95 \%$ 169-3.473), having secondary education (AOR $0.858, \mathrm{Cl} 95 \%$ 0.352-3.473), having tertiary education (AOR $0.830, \mathrm{Cl} 95 \%$ 0.263-2.621), being married (AOR 0.481, Cl 95\% 0.223-1.035), being single (AOR 0.583, Cl 95\% 0.273-1.222) and using tenofovir, lamivudine with efavirenz-TDF/3TC/ 
Citation: Obeng RK, Anto BP, Attakorah J, et al. (2021) Retention in Care, Loss to Follow-up and Associated Patient Characteristics: A Retrospective Cohort Study among Adults Receiving Antiretroviral Therapy from Urban Health Facilities in Ghana. Clin J HIV AIDS 5(1):70-75

Table 2: Logistic regression analysis results of patients retained in urban antiretroviral therapy program in Ghana.

\begin{tabular}{|c|c|c|c|c|}
\hline Patient characteristics & $\begin{array}{l}\text { Number of patients retained } \\
\text { in care }\end{array}$ & Adjusted odds ratio (AOR) & p-value & $\begin{array}{l}\text { 95\% confidence } \\
\text { interval }\end{array}$ \\
\hline \multicolumn{5}{|l|}{ Age (Years) } \\
\hline $21-40(n=200)$ & $135(67.5 \%)$ & 0.767 & 0.731 & $0.169-3.473$ \\
\hline $41-60(n=160)$ & $118(73.8 \%)$ & 1.011 & 0.989 & $0.226-4.517$ \\
\hline $61-80(n=10)$ & $7(70.0 \%)$ & 1.000 & & \\
\hline \multicolumn{5}{|l|}{ Sex } \\
\hline Female $(n=258)$ & $188(72.9 \%)$ & 1.319 & 0.332 & $0.754-2.310$ \\
\hline Male $(n=112)$ & $72(64.3 \%)$ & 1.000 & & \\
\hline \multicolumn{5}{|l|}{ Education } \\
\hline Primary $(n=119)$ & $150(75.4 \%)$ & 1.539 & 0.104 & $0.915-2.590$ \\
\hline Secondary $(n=28)$ & $16(57.1 \%)$ & 0.858 & 0.737 & $0.352-2.091$ \\
\hline Tertiary $(n=16)$ & $8(50.0 \%)$ & 0.83 & 0.751 & $0.263-2.621$ \\
\hline Uneducated $(n=127)$ & $86(67.7 \%)$ & 1.000 & & \\
\hline \multicolumn{5}{|l|}{ Marital status } \\
\hline Divorced $(n=72)$ & $60(83.3 \%)$ & 1.266 & 0.613 & $0.506-3.166$ \\
\hline Married $(n=146)$ & $91(62.3 \%)$ & 0.481 & 0.061 & $0.223-1.035$ \\
\hline Single $(n=89)$ & $59(66.3 \%)$ & 0.538 & 0.139 & $0.237-1.222$ \\
\hline Widowed $(n=63)$ & $50(79.4 \%)$ & 1.000 & & \\
\hline \multicolumn{5}{|l|}{ Occupation } \\
\hline Employed ( $n=333$ ) & 240 (72.1\%) & 2.55 & 0.015 & $1.201-5.414$ \\
\hline Unemployed $(n=37)$ & $20(54.7 \%)$ & 1.000 & & \\
\hline \multicolumn{5}{|l|}{ Regimen } \\
\hline AZT/3TC/EFV $(n=88)$ & $54(61.4 \%)$ & 0.652 & 0.276 & $0.302-1.407$ \\
\hline AZT/3TC/NVP $(n=109)$ & 79 (72.5\%) & 1.125 & 0.759 & $0.531-2.385$ \\
\hline TDF/3TC/EFV $(n=114)$ & $86(75.4 \%)$ & 1.024 & 0.95 & $0.488-2.148$ \\
\hline TDF/3TC/NVP $(n=63)$ & 42 (71.2\%) & 1.000 & & \\
\hline
\end{tabular}

Note: TDF is tenofovirdisoproxylfumarate, AZT is azidothymidine (zidovudine), 3 TC is lamivudine, NVP is nevirapine and EFV is efavirenz

Table 3: Loss to follow-up time among Ghanaian adults offered antiretroviral therapy in urban health facilities.

\begin{tabular}{|l|l|l|}
\hline $\begin{array}{l}\text { Loss to follow-up } \\
\text { time/Months }\end{array}$ & $\begin{array}{l}\text { No. of patients LTFU } \\
(\mathbf{N}=\mathbf{1 1 0})\end{array}$ & \% of patients LTFU \\
\hline$<1$ (NFUV) & 22 & 20 \\
\hline $00-06$ & 30 & 27.3 \\
\hline $07-12$ & 15 & 13.6 \\
\hline $13-18$ & 13 & 11.8 \\
\hline $19-24$ & 9 & 8.2 \\
\hline $25-30$ & 17 & 15.5 \\
\hline $31-36$ & 4 & 3.6 \\
\hline
\end{tabular}

EFV (AOR $0.652 \mathrm{Cl} 95 \%$ 0.302-1.407) were associated with reduced odds of being retained in ART up to 3-years.

Table 3 shows that out of the 110 patients who were lost to follow-up, $20 \%(n=22)$ had no follow-up visit (NFUV) after treatment initiation. In other words such patients did not return to the hospital for continuation of care after initiation of ART. Cumulatively $47.3 \%, 60.9 \%, 72.7 \%, 80.9 \%$ and $96.4 \%$ of patients were lost to follow-up within 6, 12, 18, 24 and 30 months of ART respectively.

\section{Mortality}

A total of 10 (9.1\%) deaths were recorded out of 110 patients who were lost to follow-up. Overall mortality of the study patients was therefore $2.7 \%$ within 3 -years of ART. All the 10 deaths were recorded in patients' folders. No records of cause of death were found in folders. We did not conduct verbal autopsy to ascertain the cause of death.

\section{Discussion}

In this study which we investigated retention in care, loss to follow-up and mortality among patients receiving antiretroviral therapy from urban health facilities in Ghana, the results show that being younger adult, having secondary or tertiary education, being married or single and using tenofovir, lamivudine with efavirenz (TDF/3TC/EFV) were associated with reduced odds of being retained in ART. The most significant limitations of the study were the retrospective design and incomplete documentation of some patients' details. 
Citation: Obeng RK, Anto BP, Attakorah J, et al. (2021) Retention in Care, Loss to Follow-up and Associated Patient Characteristics: A Retrospective Cohort Study among Adults Receiving Antiretroviral Therapy from Urban Health Facilities in Ghana. Clin J HIV AIDS 5(1):70-75

The overall LTFU of $29.7 \%$ within 3 years of ART found in this study is almost equal to the results of Collini, et al. 2009 who obtained $31 \%$ of LTFU from Komfo Anokye Teaching Hospital (KATH). The marginal reduction in LTFU cases may be attributed to the enhanced counseling and follow-up efforts by the clinical care providers of the two health facilities. Our retention figure of $70.3 \%$ is however higher than that estimated for Africa (65\%) by Fox \& Rosen, 2015 [5]. The results of this study show that $64.3 \%$ males as compared to $72.9 \%$ females (AOR 1.319, 95\% Cl 0.754-2.310) were retained in care (Table 2). The trend is consistent with results of other researchers $[15,16]$.

Table 1 shows that patients aged 21-40 years and 4160 years constituted $97.3 \%(n=360)$. Between the two age groups, loss to follow-up was higher (32\%) among the 21-40-years age group implying reduced odds of retention in care (AOR $0.76795 \% \mathrm{Cl} 0.169-3.373$ ) than the 41-60-years group with $26.2 \%$ LTFU and relatively higher odds of being retained in care (AOR 1.011, 95\% Cl 0.226-4.517). These results are consistent with findings of Fatti, et al. 2014 that crude cumulative incidences of loss to follow-up on ART were higher among younger adults than older ones [17]. Although patients in the $61-80$-years group also had $30 \%$ LTFU, they constituted only $2.7 \%$ of the study patients.

Regarding loss to follow-up and occupational background, the results show that employed patients (AOR 2.550, 95\% $\mathrm{Cl}$ 1.201-5.414) had higher odds of being retained in care as compared to unemployed patients who had higher LTFU of $46 \%$. This situation is expected because unemployment has strong association with socio-economic status $[18,19]$. Although antiretroviral medicines could be picked by patients from their facility pharmacies of the study sites without outof-pocket payment, financial challenges associated with transportation cost of visiting ART sites have been identified in other studies among patients of our study sites. The reduced odds of being retained in care among married patients (AOR $0.48195 \% \mathrm{Cl} 0.223-1.035$ ) as compared to the unmarried (single, divorced and widowed) could be interpreted on the basis that married clients find it difficult to disclose their HIV+ status to their sexual partners for fear of stigmatizing attitude, possible rejection and domestic violence especially among women [20,21]. Wolf, et al. 2014 have reported association between HIV-related stigma and LTFU [22].

Cumulative LTFU of $47.3 \%$ within the first six months of ART (which includes $20.7 \%$ of those who had no follow-up visits) is very alarming. These findings from our study support those of Zachariah, et al., 2006 [23] who recorded early attrition from treatment due to $12.6 \%$ deaths, of which $61 \%$ and $79 \%$ occurred within the first 3 months and 6 months of ART respectively.

Implications of the study results are worth considering. Of the 110 patients who were lost to follow-up, 10 had died leaving 100 (approximately) $27 \%$ of the study patients. The clinical, social and economic implications of $27 \%$ ART dropouts without viral suppression need to be considered. Firstly, such persons have the potential to transmit HIV to sexual partners with whom they engage in unprotected sex [24]. Secondly, women of reproductive age among this group of
ART drop-outs have the potential to transmit HIV to their babies if they become pregnant and do not receive prevention of mother to child transmission (PMTCT) interventions [25]. Thirdly, ART non-adherent patients may develop resistant HIV strains which will in turn be transmitted to other persons. Since cost of treatment of resistant HIV infection is usually higher, health budgets in settings with resistant HIV strains are likely to increase [26]. Lastly, in the context of UNAIDS' 909090 target $[27,28]$ of HIV service delivery by the year 2020 and its extension to 959595 by the year 2030 [29], the study sites may not be able to achieve the second and third targets of $95 \%$ patient retention and viral suppression unless pragmatic interventions are designed to achieve the target.

Although mortality among our study cohort was low and $70 \%$ were retained in ART up to 3-years, loss to follow-up was high especially within the first 6 months of therapy. We observed that being female, aged 41-60-years, employed and divorced were associated with increased odds of being retained in care. Effective follow-up interventions are required to reduce LTFU within the first 6 months of treatment and among younger adults, patients with secondary and tertiary education as well as single and married patients who were less likely to be retained in care.

\section{Acknowledgements}

The authors would like to acknowledge the contributions of all Chest Clinic Pharmacy staff of Komfo Anokye Teaching Hospital for their immense assistance. We would also like to acknowledge Miss Akua Afriyie of Suntreso Government Hospital for her assistance in the data collection process.

\section{Funding}

This research received no specific grant from any funding agency in the public, commercial, or not-for-profit sectors.

\section{Ethical Clearance and Consent to Participate}

This work did not require ethical clearance and consent to participate because there were no human subjects involved.

\section{Conflict of Interest}

The authors declare that there is no conflict of interest.

\section{Author Contributions}

RKO participated in the study design, data collection and analysis and drafting of manuscript; BPA participated in the study design, data analysis and also edited the manuscript; $\mathrm{JA}, \mathrm{EF}$ and RM also participated in the study design, data collection, analysis and preparation of the manuscript.

\section{References}

1. Kanters S, Vitoria M, Doherty M, et al. (2016) Comparative efficacy and safety of first-line antiretroviral therapy for the treatment of HIV infection: A systematic review and network meta-analysis. Lancet HIV 3: e510-e520.

2. Haas AD, Zaniewski E, Anderegg N, et al. (2018) Retention and mortality on antiretroviral therapy in sub-Saharan Africa: Collaborative analyses of HIV treatment programmes. J Int AIDS Soc 21: e25084. 
Citation: Obeng RK, Anto BP, Attakorah J, et al. (2021) Retention in Care, Loss to Follow-up and Associated Patient Characteristics: A Retrospective Cohort Study among Adults Receiving Antiretroviral Therapy from Urban Health Facilities in Ghana. Clin J HIV AIDS 5(1):70-75

3. Gesesew HA, Ward P, Hajito KW, et al. (2017) Discontinuation from Antiretroviral Therapy: A Continuing Challenge among Adults in HIV Care in Ethiopia: A Systematic Review and MetaAnalysis. PLoS One 12: e0169651.

4. Barth RE, Tempelman HA, Moraba R, et al. (2011) Long-Term Outcome of an HIV-Treatment Programme in Rural Africa: Viral Suppression despite Early Mortality AIDS Res Treat 2011: e434375.

5. Fox MP, MPA SR (2015) Retention of adult patients on antiretroviral therapy in low- and middle-income countries: Systematic review and meta-analysis 2008-2013. J Acquir Immune Defic Syndr 69: 98-108.

6. Tsegaye E, Worku A (2011) "Assessment of antiretroviral treatment outcome in public hospitals, South Nations Nationalities and Peoples Region, Ethiopia". Ethiop J Health Dev 25: 102-109.

7. Moges NA, Olubukola A, Micheal O, et al. (2020) "HIV patients retention and attrition in care and their determinants in Ethiopia: A systematic review and meta-analysis". BMC Infect Dis 20: 439.

8. Collini P (2009) "Sustained immunological responses to highly active antiretroviral therapy at 36 Months in a ghanaian HIV cohort. Clin Infect Dis 48: 988-991.

9. Mecha JO (2018) "Trends, treatment outcomes, and determinants for attrition among adult patients in care at a large tertiary HIV clinic in Nairobi, Kenya: A 2004-2015 retrospective cohort study". HIV AIDS (Auckl) 10: 103-114.

10. Alvarez-Uria G, Naik PK, Pakam R, et al. (2013) "Factors associated with attrition, mortality, and loss to follow up after antiretroviral therapy initiation: Data from an HIV cohort study in India". Global Health Action 6: 21682.

11. Lawn SD, Harries AD, Anglaret X, et al. (2008) Early mortality among adults accessing antiretroviral treatment programmes in sub-Saharan Africa. AIDS 22

12. Egger S, Petoumenos K, Kamarulzaman A, et al. (2006) Longterm patterns in CD4 response is determined by an interaction between baseline CD4 cell count, viral load and time: the Asia Pacific HIV Observational Database (APHOD). J Acquir Immune Defic Syndr 50: 513-520.

13. Bill G (2004) "Sample Size Formulas References". Scientific Research Publishing.

14. Rose A, Spinks N, Canhoto Al (2015) Management research, applying the principles. Routledge New York: Routledge, Taylor \& Francis Group.

15. Wandeler G, Keiser O, Pfeiffer K, et al. (2012) "Outcomes of antiretroviral treatment programs in rural Southern Africa". J Acquir Immune Defic Syndr 59: e9-e16.

16. Mutasa-Apollo T (2014) "Patient retention, clinical outcomes and attrition-associated factors of HIV-infected patients enrolled in Zimbabwe's national antiretroviral therapy programme, 20072010". PLoS One 9: 1.
17. Fatti G, Mothibi E, Meintjes G, et al. (2014) “Antiretroviral Treatment Outcomes amongst Older Adults in a Large Multicentre Cohort in South Africa". PLoS One 9: e100273.

18. Tran BX, Hwang J, Nguyen LH, et al. (2016) "Impact of Socioeconomic Inequality on access, adherence, and outcomes of antiretroviral treatment services for people living with HIV/ AIDS in Vietnam". PLoS One 11: e0168687.

19. Moomba K, van Wyk B (2019) "Social and economic barriers to adherence among patients at Livingstone General Hospital in Zambia". Afr J Prim Health Care Fam Med 11: e1-e6.

20. Buregyeya E, Naigino R, Mukose A, et al. (2017) "Facilitators and barriers to uptake and adherence to lifelong antiretroviral therapy among HIV infected pregnant women in Uganda: A Qualitative Study". BMC Pregnancy and Childbirth 17: 94.

21. Maeri I, El Ayadi A, Getahun M, et al. (2016) “'How can I tell?' Consequences of HIV status disclosure among couples in eastern African communities in the context of an ongoing HIV 'test-andtreat' trial. AIDS Care 28: 59-66.

22. Wolf HT, Halpern-Felsher BL, Bukusi EA, et al. (2014) It is all about the fear of being discriminated [against]...the person suffering from HIV will not be accepted': A qualitative study exploring the reasons for loss to follow-up among HIV-positive youth in Kisumu, Kenya. BMC Public Health 14: 1154.

23. Zachariah R, Fitzgerald M, Massaquoi M, et al. (2006) Risk factors for high early mortality in patients on antiretroviral treatment in a rural district of Malawi. AIDS 20: 2355-2360.

24. Rodger AJ, Cambiano V, Bruun T, et al. (2016) Sexual activity without condoms and risk of HIV transmission in serodifferent couples when the HIV-positive partner is using suppressive antiretroviral therapy. JAMA 316: 171-181.

25. Bokharaei-Salim F, Kalantari S, Gholamypour Z, et al. (2018) Investigation of the effects of a prevention of mother-to-child HIV transmission program among Iranian neonates. Arch Virol 163: 1179-1185.

26. Snedecor SJ, Khachatryan A, Nedrow K, et al. (2013) The prevalence of transmitted resistance to first-generation nonnucleoside reverse transcriptase inhibitors and its potential economic impact in HIV-infected patients. PLoS One 8: e72784.

27. ht t ps://www.unaids.org/en/resources/ documents/2017/90-90-90

28. Bain LE, Nkoke C, Noubiap JJN (2017) UNAIDS 90-90-90 targets to end the AIDS epidemic by 2020 are not realistic: Comment on 'Can the UNAIDS 90-90-90 target be achieved? A systematic analysis of national HIV treatment cascades. BMJ Glob Health 2: e000227.

29. (2014) UNAIDS Issues New Fast-Track Strategy to End AIDS by 2030 - EGPAF. Elizabeth Glaser Pediatric AIDS Foundation.

DOI: $10.36959 / 695 / 574$

Copyright: (c) 2021 Fenkl EA, et al. This is an open-access article distributed under the terms of the Creative Commons Attribution License, which permits unrestricted use, distribution, and reproduction in any medium, provided the original author and source are credited. 